\title{
Spatiotemporal patterns in a general networked activator-substrate model
}

\author{
Mengxin Chen - Qianqian Zheng - Ranchao Wu - Liping Chen
}

\begin{abstract}
For the purpose of understanding the spatiotemporal pattern formation in the random networked system, a general activator-substrate model with network structure is introduced. Firstly, we investigate the boundedness of the non-constant steady state of the elliptic system of the continuous media system. It is found that the non-constant steady state admits their upper and lower bounds with certain conditions. Then, one investigates some properties and non-existence of the non-constant steady state with the no-flux boundary conditions. The main results show that the diffusion rate of activator should greater than the diffusion rate of substrate. Otherwise, there might be no pattern formation of the system. Afterwards, a general random networked activator-substrate model is made public. The conditions of the stability, the Hopf bifurcation, the Turing instability and a co-dimensional-two TuringHopf bifurcation are yield by the method of stability analysis and bifurcation theorem. Finally, we choose a suitable sub-system of the general activator-substrate model to verify the theoretical results, and full numerical simulations are well verified these results. Especially, an interesting finding is that the stability of the positive equilibrium will switch from unstable to stable one
\end{abstract}

M.X. Chen

College of Mathematics and Information Science, Henan Normal University, Xinxiang, 453007, China

Q.Q. Zheng

School of Mathematics and Statistics, Xuchang University, Xuchang, 461000, China

R.C. $\mathrm{Wu}(\square)$

School of Mathematical Sciences, Anhui University, Hefei 230601, China

E-mail: rcwu@ahu.edu.cn

L.P Chen

School of Electrical Engineering and Automation, Hefei University of Technology, Hefei 230009, China with the change of the connection probability of the nodes, this is different from the pattern formation in the continuous media systems.

Keywords Activator-substrate model, Random network, Spatiotemporal patterns, Non-constant steady state.

\section{Introduction}

In the real world, reaction-diffusion is one of the most basic movement processes, and it can be used to describe the spread of diseases, chemical reactions, biological migration and other phenomena. Hence, many coupled reaction-diffusion equations are used to understand this process in a continuous space. An interesting and challenging research area is understanding the Turing patterns by a coupled reaction-diffusion equation [1-4]. Along this way, there has been a great deal of accumulated achievements to enrich pattern formations within coupled reaction-diffusion systems. Such as, the stripe patterns, the stripe and the spotted mixed patterns in a one-dimensional region $[5,6]$, and more complex hexagonal patterns, the labyrinthine-like stripe patterns, Eckhaus patterns, chaos in a two-dimensional region $[7,8]$. What is more, the spatiotemporal patterns also have been investigated where the Turing mode and the non-Turing mode intersect by reaction-diffusion equations. For instance, a codimensional-two TuringHopf bifurcation was considered in $[9,10]$ by the technique of the normal form theory and the multiple time scales, respectively.

It is noticed that models in the references mentioned above are governed by the continuous systems. However, as it is known that in many cases the reactiondiffusion process under scrutiny is defined in a discrete 
media rather than in a continuous one, such as disease spread and control, population dynamics and so on. Therefore, could such rich spatial and spatiotemporal behaviors happen in the discrete reaction-diffusion systems? Keep all in mind, a reaction-diffusion model with the network structure may be worth considering. Asllani et al. [11] showed that the homogeneous fixed point can become unstable due to the topology of the network, which cannot be induced on undirected graphs, and the traveling waves, or quasi-stationary patterns can be found in a network system. Diego et al. [12] provided a first general theory of Turing network topology, which proves why three key features of a Turing system are directly determined by the topology, and they also yield that in experimental systems, it is easier to obtain reliable information about the topology of a network than precise values of diffusion constants. The instability and pattern formations on several different networks including deterministic networks and random networks with time delay are explored by Chang et al. [13], and wave patterns can be generated on different networks by numerical simulations. The Hopf bifurcation and its detailed nature have been considered in a network-organized semiarid vegetation model with time delay by Tian et al. in [14]. For more dynamical results on the network-organized systems one could refer to Refs. [15-18] and references cited therein.

However, we note that the results about the influence of the connection probability of nodes are few [19]. Inspired by this discovery, we will explore the influence of the connection probability of nodes in a networked model. To this end, we first introduce a continuous media system of the form

$$
\begin{cases}\frac{\partial u}{\partial t}-d_{1} \Delta u=u^{k} f(v)-\beta u, & x \in \Omega, t>0, \\ \frac{\partial v}{\partial t}-d_{2} \Delta v=c-u^{k} f(v)-v, & x \in \Omega, t>0, \\ \frac{\partial u}{\partial \mathbf{n}}=\frac{\partial v}{\partial \mathbf{n}}=0, & x \in \partial \Omega, t \geq 0, \\ u(x, 0)=u_{0}(x) \geq 0, & x \in \Omega, \\ v(x, 0)=v_{0}(x) \geq 0, & \end{cases}
$$

where $u=u(x, t)$ and $v=v(x, t)$ are the concentrations of the activator and the substrate, respectively; constants $d_{1}$ and $d_{2}$ stand for the diffusion coefficients of activator $u$ and substrate $v$, respectively; $k \geq 2$ and $f(v)$ is a monotone increasing function with respect to $v$; spatial domain $\Omega$ is a bounded domain in $R^{n}(n \geq 1)$ with the boundary $\partial \Omega ; \mathbf{n}$ is the outward unit normal vector along $\partial \Omega$ and $\partial \mathbf{n}$ represents the operator of directional derivative along the direction $\mathbf{n}$; parameters $c, \beta, d_{1}, d_{2}$ are positive constants from the point of view of chemistry. The dimensionless system (1) is commonly called the depletion model or the activator-substrate model, and it firstly proposed by Gierer and Meinhardt in 1972 [20]. Meinhardt and Klingler [21] employed this model to explore pigmentation and relief-like patterns on mollusc shells when $f(v)=v$ and replaced $u^{k}$ by $\frac{u^{2}}{1+\kappa u^{2}}+\rho_{0}$, where $\kappa>0$ and $\rho_{0}$ represents a small base production of the activator. A similar results has been carried out by Fowler et al. [22] to describe the pigmentation patterns on shell surfaces. The Hopf bifurcation and its direction as well as the Turing instability have been studied by $\mathrm{Wu}$ et al. [23] when the monotone increasing function $f(v)=v$ and $k=2$. Also, the asymptotic stability of constant steady states, the steady state bifurcations from constant steady states are analyzed both in one-dimensional kernel and in two-dimensional kernel cases have been investigated by Wang et al. in [24]. For more results on activator-substrate model (1) one can refer to Refs. [25,28].

Now, if we assume that each node of the network owns the homogeneous densities in a small community, a general networked activator-substrate model takes the form:

$$
\left\{\begin{array}{l}
\frac{d u_{i}}{d t}-d_{1} \sum_{j=1}^{N} \Delta_{i j} u_{i}=u_{i}^{k} f\left(v_{i}\right)-\beta u_{i} \\
\frac{d v_{i}}{d t}-d_{2} \sum_{j=1}^{N} \Delta_{i j} v_{i}=c-u_{i}^{k} f\left(v_{i}\right)-v_{i}
\end{array}\right.
$$

Here, we assume that the networked model (2) is defined on an undirected network with $N$ nodes and there is no self-loops; $u_{i}$ and $v_{i}$ are the concentrations of the activator and the substrate on node $i$, respectively; $\boldsymbol{\Delta}$ is the $N \times N$ Laplacian matrix of network set to be $\Delta_{i j}=k_{i} \delta_{i j}-L_{i j}$, at which $\mathbf{L}$ is the adjacency matrix encoding the network connection, namely it satisfies $L_{i j}=1$ if there is a link connecting from patch $i$ to patch $j$, otherwise, $L_{i j}=0$ if there is no any link connecting from patch $i$ to patch $j$, and $\delta_{i j}$ is the Kronecker's delta. In addition, the degree of the $i$ th node is defined by $k_{i}=\sum_{j=1}^{N} L_{i j}$, and $p(0 \leq p \leq 1)$ is set to be the connection probability between node $i$ and node $j$ for $i \neq j$.

In this work, we shall research the dynamics of the general activator-substrate model (1) and networked model (2), respectively. More precisely, the boundedness of the positive non-constant steady state will be studied for the elliptic system of the continuous general activator-substrate model (1). With the help of the maximum principle and the Harnack's inequality one shows that it admits the upper bounds and the lower bounds for any positive solution $(u(x), v(x))$ under certain conditions. Also, we explore the sufficient conditions to ensure the non-existence of the non-constant steady state by means of the Poincaré's inequality and Cauchy-Schwarz inequality. It is found that there is no non-constant steady state of the elliptic system if 
the diffusion rate of activator greater than the diffusion rate of substrate. This is very useful information for studying the formation of spatiotemporal patterns. Moreover, we would like to mention that all the results obtained above employ the homogeneous Neumann boundary conditions and assume that $f(v)$ satisfies $\left(C_{0}\right)$, where

$$
\begin{array}{r}
\left(C_{0}\right): f \in C^{1}(0, \infty) \cap C[0, \infty), f(0)=0, f(v)>0 \\
\text { and } \delta>f^{\prime}(v)>0 \text { for } v \in(0, \infty) .
\end{array}
$$

For the networked activator-substrate model (2), some general results of the existence of internal positive equilibrium and its stability, the Hopf bifurcation and the Turing-Hopf bifurcation are given, and a special example is considered to verify these general results. As we know, the Turing-Hopf bifurcation is a codimensional-two bifurcation, at which both the Hopf mode and the Turing mode will intersect. Hence, the formation of patterns may more complex than patterns near the Hopf mode and the Turing mode. Compared with the works done in [14] and references cited therein, this work has two highlights: (i) In addition to the Hopf bifurcation and the Turing instability, the spatiotemporal patterns near the Turing-Hopf bifurcation is also considered by theoretically and numerically. (ii) The numerical simulation results indicate that the connection probability $p$ has a crucial influence on the Turing pattern formation, namely the stability of the internal positive equilibrium will switch from unstable to stable one with the changing of the connection probability $p$, although we fix the parameters of model (2) in the Turing instability region.

The layout of this paper is structured as follows. In Sect. 2, the boundedness and the non-existence of the non-constant steady state of the related elliptic system (1) are carried out. In Sect. 3, stability, existence of the Hopf bifurcation, the Turing instability and the TuringHopf bifurcation of the general networked activatorsubstrate model (2) are considered. Then, a sub-system of the general networked activator-substrate model (2) is introduced in Sect. 4, and full numerical simulations are finished in Sect. 5 to verify that the theoretical reality. Finally, some discussions and conclusions are made in Sect. 6.

\section{Non-constant steady state}

In this section, we shall investigate the non-existence of the non-constant steady state of the elliptic system, it is governed by

$$
\begin{cases}d_{1} \Delta u(x)+u^{k}(x) f(v(x))-\beta u(x)=0, & x \in \Omega, \\ d_{2} \Delta v(x)+c-u^{k}(x) f(v(x))-v(x)=0, & x \in \Omega, \\ \frac{\partial u}{\partial \mathbf{n}}=\frac{\partial v}{\partial \mathbf{n}}=0, & x \in \partial \Omega .\end{cases}
$$

\subsection{A priori estimates}

Lemma 1 ([26]) Suppose that $F(x, \omega(x)) \in C(\bar{\Omega} \times R)$. (i) If $\omega(x) \in C^{2}(\Omega) \cap C^{1}(\bar{\Omega})$ satisfies

$$
\begin{cases}\Delta \omega(x)+F(x, \omega(x)) \geq 0, & \text { in } \Omega, \\ \frac{\partial \omega(x)}{\partial \mathbf{n}} \leq 0 & \text { on } \partial \Omega\end{cases}
$$

and $\omega\left(x_{0}\right)=\max _{x \in \bar{\Omega}} \omega(x)$, then $F\left(x_{0}, \omega\left(x_{0}\right)\right) \geq 0$.

(ii) If $\omega(x) \in C^{2}(\Omega) \cap C^{1}(\bar{\Omega})$ satisfies

$$
\begin{cases}\Delta \omega(x)+F(x, \omega(x)) \leq 0, & \text { in } \Omega, \\ \frac{\partial \omega(x)}{\partial \mathbf{n}} \geq 0 & \text { on } \partial \Omega\end{cases}
$$

and $\omega\left(x_{0}\right)=\min _{x \in \bar{\Omega}} \omega(x)$, then $F\left(x_{0}, \omega\left(x_{0}\right)\right) \leq 0$.

Lemma 2 ([27]) Suppose that $\omega(x) \in C^{2}(\Omega) \cap C^{1}(\bar{\Omega})$ be a positive solution of

$$
\Delta \omega(x)+c(x) \omega(x)=0, x \in \Omega, \frac{\partial \omega(x)}{\partial \mathbf{n}}=0 \text { on } \partial \Omega,
$$

and satisfying homogeneous no-flux boundary conditions and $c(x) \in C(\Omega) \cap L^{\infty}(\Omega)$. Then there exists a positive constant $c_{*}=c_{*}\left(\|c(x)\|_{\infty}, \Omega\right)$ such that

$\max _{x \in \bar{\Omega}} \omega(x) \leq c_{*} \min _{x \in \bar{\Omega}} \omega(x)$.

Theorem 1 Suppose that $c, \beta, d_{1}$ and $d_{2}$ are positive constants, $k \geq 2$ and $f$ satisfies condition $\left(C_{0}\right)$. If $(u(x), v(x))$ is a positive solution of system (3), then there is a constant $\sigma>0$ such that $\|f\|_{\infty} \leq \sigma$ and

$$
\frac{1}{c_{*}}\left(\frac{\beta}{\sigma}\right)^{1-k} \leq u(x) \leq c \max \left\{\frac{d_{2}}{d_{1}}, \frac{1}{\beta}\right\},
$$

and

$c-\sigma c^{k}\left(\max \left\{\frac{d_{2}}{d_{1}}, \frac{1}{\beta}\right\}\right)^{k} \leq v(x) \leq c$,

where positive constant $c_{*}$ only depends on

$$
\begin{aligned}
& \left\|\frac{1}{d_{1}}\left(u^{k}(x) f(v(x))-\beta u(x)\right)\right\|_{\infty} \\
& \leq \frac{1}{d_{1}}\left(\sigma c^{k}\left(\max \left\{\frac{d_{2}}{d_{1}}, \frac{1}{\beta}\right\}\right)^{k}+\beta c \max \left\{\frac{d_{2}}{d_{1}}, \frac{1}{\beta}\right\}\right) .
\end{aligned}
$$


Proof. We assume that $(u(x), v(x))$ is a positive solution of system (3), and

$$
\begin{array}{r}
u\left(x_{0}\right)=\min _{x \in \bar{\Omega}} u(x), \quad u\left(x_{1}\right)=\max _{x \in \bar{\Omega}} u(x), \\
v\left(y_{0}\right)=\min _{x \in \bar{\Omega}} v(x) \quad v\left(y_{1}\right)=\max _{x \in \bar{\Omega}} v(x) .
\end{array}
$$

By Lemma 1 and the second equation of (3), we have $c-u^{k}\left(y_{1}\right) f\left(v\left(y_{1}\right)\right)-v\left(y_{1}\right) \geq 0$,

this leads to $v\left(y_{1}\right) \leq c$, namely $v(x) \leq c$ for $x \in \bar{\Omega}$. Noticing that function $f(v(x))$ satisfies condition $\left(C_{0}\right)$ and $v(x) \leq c$ for $x \in \bar{\Omega}$, we claim that there is a positive constant $\sigma>0$ such that $\|f(v(x))\|_{\infty} \leq \sigma$ holds for $x \in \bar{\Omega}$. As a result, by the first equation of (3), we have

$u^{k}\left(x_{1}\right) f\left(v\left(x_{1}\right)\right)-\beta u\left(x_{1}\right) \geq 0$,

it leads to a fact $u\left(x_{1}\right) \geq\left(\frac{\beta}{\sigma}\right)^{1-k}$. form

On the other hand, defining a linear function of the

$\omega(x)=d_{1} u(x)+d_{2} v(x)$,

it follows that

$\Delta \omega(x)=\beta u(x)+v(x)-c, \quad x \in \Omega, \quad \frac{\partial \omega}{\partial \mathbf{n}}=0$, on $\partial \Omega$.

In addition, we assume that $\omega(x)$ has maximum at $x=z_{1}$, i.e., $\omega\left(z_{1}\right)=\max _{x \in \bar{\Omega}} \omega(x)$. Then by means of Lemma 1 and Eqs. (6), (7), one obtains

$u\left(z_{1}\right) \leq \frac{1}{\beta}\left(c-v\left(z_{1}\right)\right), \quad \omega\left(z_{1}\right) \leq \frac{c d_{1}}{\beta}+\left(d_{2}-\frac{d_{1}}{\beta}\right) v\left(z_{1}\right)$.

Consequently, we have the following result

$d_{1} u\left(x_{1}\right) \leq \omega\left(x_{1}\right) \leq \omega\left(z_{1}\right) \leq \frac{c d_{1}}{\beta}+\left(d_{2}-\frac{d_{1}}{\beta}\right) v\left(z_{1}\right)$.

It is noticed that $v(x) \leq c$, then one has

$u\left(x_{1}\right) \leq \frac{c}{\beta}+c\left(\frac{d_{2}}{d_{1}}-\frac{1}{\beta}\right) \leq c \max \left\{\frac{d_{2}}{d_{1}}, \frac{1}{\beta}\right\}$,

this indicates that $u(x) \leq c \max \left\{\frac{d_{2}}{d_{1}}, \frac{1}{\beta}\right\}$ for $x \in \bar{\Omega}$.

Let

$$
c(x)=\frac{1}{d_{1}}\left(u^{k}(x) f(v(x))-\beta u(x)\right),
$$

then it is easy to check out $\|c(x)\|_{\infty} \leq M$, where

$M=$

$\frac{1}{d_{1}}\left(\sigma c^{k}\left(\max \left\{\frac{d_{2}}{d_{1}}, \frac{1}{\beta}\right\}\right)^{k}+\beta c \max \left\{\frac{d_{2}}{d_{1}}, \frac{1}{\beta}\right\}\right)>0$.
Therefore, by Lemma 2 we claim that there is a constant $c_{*}=c_{*}\left(\|c(x)\|_{\infty}\right)>0$ such that

$\left(\frac{\beta}{\sigma}\right)^{1-k} \leq u\left(x_{1}\right) \leq c_{*} u\left(x_{0}\right)$,

it then follows that $u\left(x_{0}\right) \geq \frac{1}{c_{*}}\left(\frac{\beta}{\sigma}\right)^{1-k}$, namely $u(x) \geq$ $\frac{1}{c_{*}}\left(\frac{\beta}{\sigma}\right)^{1-k}$ for $x \in \bar{\Omega}$.

By employing Lemma 1 and the second equation of (3) again, we have

$c-u^{k}\left(y_{0}\right) f\left(v\left(y_{0}\right)\right)-v\left(y_{0}\right) \leq 0$,

this leads to

$v\left(y_{0}\right) \geq c-\sigma c^{k}\left(\max \left\{\frac{d_{2}}{d_{1}}, \frac{1}{\beta}\right\}\right)^{k}$.

The proof is completed.

In what follows, we shall research non-existence of the non-constant steady state solution of system (3). To do so, let $0=\lambda_{0}<\lambda_{1}<\lambda_{2}<\cdots<\lambda_{i}<\cdots$ and $\lim _{i \rightarrow \infty} \lambda_{i}=\infty$ be the complete set of eigenvalues of the operator $-\Delta$ with no-flux boundary conditions in $\Omega$.

\subsection{Non-existence of non-constant steady state}

Suppose that $(u(x), v(x))$ is a positive solution of system (3), we denote their averages over domain $\Omega$ by

$\bar{u}=\frac{1}{|\Omega|} \int_{\Omega} u(x) d x$ and $\bar{v}=\frac{1}{|\Omega|} \int_{\Omega} v(x) d x$.

Then it is easy to verify a fact that

$\int_{\Omega}(u-\bar{u}) d x=0$ and $\int_{\Omega}(v-\bar{v}) d x=0$.

Let $\phi=u-\bar{u}$ and $\varphi=v-\bar{v}$, then we know that $\int_{\Omega} \phi d x=0$ and $\int_{\Omega} \varphi d x=0$.

Lemma 3 Suppose that $c, \beta, d_{1}$ and $d_{2}$ are positive constants. If $(u(x), v(x))$ is a positive solution of system (3), then $\int_{\Omega} \phi \varphi d x<0$.

Proof. From (7), we know that $\Delta \omega(x)=\beta u(x)+$ $v(x)-c$, then a straightforward computation shows that 
Spatiotemporal patterns in a general networked activator-substrate model

5

$\Delta \omega(x)=\beta \phi+\varphi$. Then multiplying it by (6) and inte- and grating by parts, one obtains

$$
\begin{aligned}
-\int_{\Omega}|\nabla \omega|^{2} d x= & \int_{\Omega}(\beta \phi+\varphi)\left(d_{1} u+d_{2} v\right) d x \\
= & \beta d_{1} \int_{\Omega} \phi u d x+\beta d_{2} \int_{\Omega} \phi v d x \\
& +d_{1} \int_{\Omega} \varphi u d x+d_{2} \int_{\Omega} \varphi v d x \\
= & \beta d_{1} \int_{\Omega} \phi^{2} d x+\beta d_{2} \int_{\Omega} \phi \varphi d x \\
& +d_{1} \int_{\Omega} \varphi \phi d x+d_{2} \int_{\Omega} \varphi^{2} d x \\
= & \beta d_{1} \int_{\Omega} \phi^{2} d x+\left(\beta d_{2}+d_{1}\right) \int_{\Omega} \phi \varphi d x \\
& +d_{2} \int_{\Omega} \varphi^{2} d x .
\end{aligned}
$$

Since $\int_{\Omega} \phi^{2} d x>0$ and $\int_{\Omega} \varphi^{2} d x>0$, this follows that $\int_{\Omega} \phi \varphi d x<0$.

Lemma 4 Suppose that $c, \beta, d_{1}$ and $d_{2}$ are positive constarts. If $(u(x), v(x))$ is a positive solution of system (3), then it admits

(i) $\int_{\Omega} \phi^{2} d x+\int_{\Omega}|\nabla \phi|^{2} d x \leq$

$\frac{\left(1+\lambda_{1}\right)|\Omega|\left(\sigma c^{k}\left(\max \left\{\frac{d_{2}}{d_{1}}, \frac{1}{\beta}\right\}\right)^{k}+\beta c \max \left\{\frac{d_{2}}{d_{1}}, \frac{1}{\beta}\right\}\right)^{2}}{d_{1}^{2} \lambda_{1}^{2}}$,

(ii) $\int_{\Omega} \varphi^{2} d x+\int_{\Omega}|\nabla \varphi|^{2} d x \leq$

$\frac{\left(1+\lambda_{1}\right)|\Omega|\left(2 c+\sigma c^{k}\left(\max \left\{\frac{d_{2}}{d_{1}}, \frac{1}{\beta}\right\}\right)^{k}\right)^{2}}{d_{2}^{2} \lambda_{1}^{2}}$.

Proof. Assume that $(u(x), v(x))$ is an arbitrary positive solution of system (3), then by Cauchy-Schwarz inequality and integrate by parts, one obtains

$$
\begin{aligned}
& d_{1} \int_{\Omega}|\nabla \phi|^{2} d x \\
= & \int_{\Omega} \phi\left(u^{k} f(v)-\beta u\right) d x \\
\leq & \left(\sigma c^{k}\left(\max \left\{\frac{d_{2}}{d_{1}}, \frac{1}{\beta}\right\}\right)^{k}+\beta c \max \left\{\frac{d_{2}}{d_{1}}, \frac{1}{\beta}\right\}\right) \\
& \times \int_{\Omega}|\phi| d x \\
\leq & \sqrt{|\Omega|}\left(\sigma c^{k}\left(\max \left\{\frac{d_{2}}{d_{1}}, \frac{1}{\beta}\right\}\right)^{k}+\beta c \max \left\{\frac{d_{2}}{d_{1}}, \frac{1}{\beta}\right\}\right) \\
& \times\left(\int_{\Omega}|\phi|^{2} d x\right)^{\frac{1}{2}},
\end{aligned}
$$

$$
\begin{aligned}
& d_{2} \int_{\Omega}|\nabla \varphi|^{2} d x \\
= & \int_{\Omega} \varphi\left(c-u^{k} f(v)-v\right) d x \\
\leq & \left(2 c+\sigma c^{k}\left(\max \left\{\frac{d_{2}}{d_{1}}, \frac{1}{\beta}\right\}\right)^{k}\right) \\
& \times \int_{\Omega}|\varphi| d x \\
\leq & \sqrt{|\Omega|}\left(2 c+\sigma c^{k}\left(\max \left\{\frac{d_{2}}{d_{1}}, \frac{1}{\beta}\right\}\right)^{k}\right) \\
& \times\left(\int_{\Omega}|\varphi|^{2} d x\right)^{\frac{1}{2}} .
\end{aligned}
$$

By employing the Poincaré's inequality, that is

$\int_{\Omega} \phi^{2} d x \leq \frac{1}{\lambda_{1}} \int_{\Omega}|\nabla \phi|^{2} d x$

$\int_{\Omega} \varphi^{2} d x \leq \frac{1}{\lambda_{1}} \int_{\Omega}|\nabla \varphi|^{2} d x$

where $\lambda_{1}$ is the first positive eigenvalue of $-\Delta$ over domain $\Omega$ with respect to the no-flux boundary conditons, we therefore obtain

$$
\begin{aligned}
& d_{1} \int_{\Omega}|\nabla \phi|^{2} d x \\
\leq & \sqrt{|\Omega|}\left(\sigma c^{k}\left(\max \left\{\frac{d_{2}}{d_{1}}, \frac{1}{\beta}\right\}\right)^{k}+\beta c \max \left\{\frac{d_{2}}{d_{1}}, \frac{1}{\beta}\right\}\right) \\
& \times\left(\int_{\Omega}|\phi|^{2} d x\right)^{\frac{1}{2}}, \\
\leq & \sqrt{\frac{|\Omega|}{\lambda_{1}}}\left(\sigma c^{k}\left(\max \left\{\frac{d_{2}}{d_{1}}, \frac{1}{\beta}\right\}\right)^{k}+\beta c \max \left\{\frac{d_{2}}{d_{1}}, \frac{1}{\beta}\right\}\right) \\
& \times\left(\int_{\Omega}|\nabla \phi|^{2} d x\right)^{\frac{1}{2}},
\end{aligned}
$$

and

$$
\begin{aligned}
& d_{2} \int_{\Omega}|\nabla \varphi|^{2} d x \\
\leq & \sqrt{|\Omega|}\left(2 c+\sigma c^{k}\left(\max \left\{\frac{d_{2}}{d_{1}}, \frac{1}{\beta}\right\}\right)^{k}\right) \\
& \times\left(\int_{\Omega}|\varphi|^{2} d x\right)^{\frac{1}{2}} \\
\leq & \sqrt{\frac{|\Omega|}{\lambda_{1}}}\left(2 c+\sigma c^{k}\left(\max \left\{\frac{d_{2}}{d_{1}}, \frac{1}{\beta}\right\}\right)^{k}\right) \\
& \times\left(\int_{\Omega}|\nabla \varphi|^{2} d x\right)^{\frac{1}{2}} \cdot
\end{aligned}
$$


This leads to

$\int_{\Omega}|\nabla \phi|^{2} d x$
$\leq \frac{|\Omega|\left(\sigma c^{k}\left(\max \left\{\frac{d_{2}}{d_{1}}, \frac{1}{\beta}\right\}\right)^{k}+\beta c \max \left\{\frac{d_{2}}{d_{1}}, \frac{1}{\beta}\right\}\right)^{2}}{d_{1}^{2} \lambda_{1}}$,

and

$\int_{\Omega}|\nabla \psi|^{2} d x \leq \frac{|\Omega|\left(2 c+\sigma c^{k}\left(\max \left\{\frac{d_{2}}{d_{1}}, \frac{1}{\beta}\right\}\right)^{k}\right)^{2}}{d_{2}^{2} \lambda_{1}}$.

By employing the Poincaré's inequality again, one achieves

$\int_{\Omega} \phi^{2} d x+\int_{\Omega}|\nabla \phi|^{2} d x$

$\left(1+\lambda_{1}\right)|\Omega|\left(\sigma c^{k}\left(\max \left\{\frac{d_{2}}{d_{1}}, \frac{1}{\beta}\right\}\right)^{k}+\beta c \max \left\{\frac{d_{2}}{d_{1}}, \frac{1}{\beta}\right\}\right)^{2} d_{1} \int_{\Omega}|\nabla \phi|^{2} d x=\int_{\Omega}\left(u^{k} f(v)-\beta u\right) \phi d x$

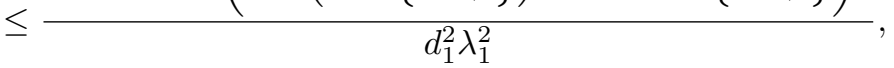

$\int_{\Omega} \psi^{2} d x+\int_{\Omega}|\nabla \psi|^{2} d x$

$\leq \frac{\left(1+\lambda_{1}\right)|\Omega|\left(2 c+\sigma c^{k}\left(\max \left\{\frac{d_{2}}{d_{1}}, \frac{1}{\beta}\right\}\right)^{k}\right)^{2}}{d_{2}^{2} \lambda_{1}^{2}}$.

This completes the proof.

Now, we consider non-existence results of the nonconstant steady state solution of system (3).

Lemma 5 Assume that $c, \beta, d_{1}$ and $d_{2}$ are positive constants in (3), then

$u^{k}-\bar{u}^{k} \leq k c^{k}\left(\max \left\{\frac{d_{2}}{d_{1}}, \frac{1}{\beta}\right\}\right)^{k}(u-\bar{u})$

Proof. Since $u(x) \leq c \max \left\{\frac{d_{2}}{d_{1}}, \frac{1}{\beta}\right\}$ for $x \in \bar{\Omega}$, then we have $\bar{u}(x) \leq c \max \left\{\frac{d_{2}}{d_{1}}, \frac{1}{\beta}\right\}$ for $x \in \bar{\Omega}$. Accordingly,

$$
\begin{aligned}
u^{k}-\bar{u}^{k} & =(u-\bar{u})\left(u^{k-1}+\bar{u} u^{k-2}+\cdots \bar{u}^{k-2} u+\bar{u}^{k-1}\right) \\
& \leq k c^{k-1}\left(\max \left\{\frac{d_{2}}{d_{1}}, \frac{1}{\beta}\right\}\right)^{k-1}(u-\bar{u}) .
\end{aligned}
$$

Now we yield the following result.

Theorem 2 Suppose that $c, \beta, d_{1}, d_{2}, k \geq 2$ are positive constants in (3) and $\Omega \subset R^{n}$ is a bounded domain with sufficient smooth boundary, then elliptic system (3) has no non-constant steady state solution if there is a positive constant $d^{*}=d^{*}(c, k, \sigma, \beta, \delta, \Omega)$ such that $\min \left\{d_{1}, d_{2}\right\}>\max \left\{d_{1}^{*}, d_{2}^{*}\right\}$, where we denote

$$
\begin{aligned}
d_{1}^{*}= & \frac{k \sigma c^{k-1}\left(\max \left\{\frac{d_{2}}{d_{1}}, \frac{1}{\beta}\right\}\right)^{k-1}}{4 \lambda_{1}} \\
& \times\left[4+k \sigma c^{k-1}\left(\max \left\{\frac{d_{2}}{d_{1}}, \frac{1}{\beta}\right\}\right)^{k-1}\right], \\
d_{2}^{*}= & \frac{c^{2 k} \delta^{2}\left(\max \left\{\frac{d_{2}}{d_{1}}, \frac{1}{\beta}\right\}\right)^{2 k}}{4 \beta \lambda_{1}} .
\end{aligned}
$$

Proof. Let $(u(x), v(x))$ be a non-negative steady state solution of system (3). Then, multiplying $\phi$ to the first equation of (3) and integrating by parts over domain $\Omega$, one yields

$$
\begin{aligned}
= & \left.\int_{\Omega}\left(u^{k} f(v)-\beta u\right)\right) \phi d x \\
& -\int_{\Omega}\left(\bar{u}^{k} f(\bar{v})-\beta \bar{u}\right) \phi d x \\
= & \int_{\Omega}\left(u^{k} f(v)-\bar{u}^{k} f(\bar{v})\right) \phi d x \\
& -\beta \int_{\Omega}(u-\bar{u}) \phi d x, \\
= & I_{1}+I_{2},
\end{aligned}
$$

where we denote

$$
\begin{aligned}
& I_{1}=\int_{\Omega}\left(u^{k} f(v)-\bar{u}^{k} f(\bar{v})\right) \phi d x, \\
& I_{2}=-\beta \int_{\Omega}(u-\bar{u}) \phi d x=-\beta \int_{\Omega} \phi^{2} d x .
\end{aligned}
$$

By Lemma 5, one has

$$
\begin{aligned}
I_{1}= & \int_{\Omega}\left(u^{k} f(v)-\bar{u}^{k} f(\bar{v})\right) \phi d x \\
= & \int_{\Omega}\left(u^{k} f(v)-\bar{u}^{k} f(v)+\bar{u}^{k} f(v)-\bar{u}^{k} f(\bar{v})\right) \phi d x \\
= & \int_{\Omega}\left(u^{k}-\bar{u}^{k}\right) f(v) \phi d x+\int_{\Omega} \bar{u}^{k}(f(v)-f(\bar{v})) \phi d x \\
\leq & k \sigma c^{k-1}\left(\max \left\{\frac{d_{2}}{d_{1}}, \frac{1}{\beta}\right\}\right)^{k-1} \int_{\Omega} \phi^{2} d x \\
& +c^{k} \delta\left(\max \left\{\frac{d_{2}}{d_{1}}, \frac{1}{\beta}\right\}\right)^{k} \int_{\Omega}|\phi \varphi| d x .
\end{aligned}
$$


Hence, the Poincaré's inequality and Cauchy-Schwarz inequality reveal that

$$
\begin{aligned}
& d_{1} \int_{\Omega}|\nabla \phi|^{2} d x \\
\leq & k \sigma c^{k-1}\left(\max \left\{\frac{d_{2}}{d_{1}}, \frac{1}{\beta}\right\}\right)^{k-1} \int_{\Omega} \phi^{2} d x \\
& +c^{k} \delta\left(\max \left\{\frac{d_{2}}{d_{1}}, \frac{1}{\beta}\right\}\right)^{k} \int_{\Omega}|\phi \varphi| d x-\beta \int_{\Omega} \phi^{2} d x \\
\leq & k \sigma c^{k-1}\left(\max \left\{\frac{d_{2}}{d_{1}}, \frac{1}{\beta}\right\}\right)^{k-1} \int_{\Omega} \phi^{2} d x \\
& +\frac{c^{2 k} \delta^{2}\left(\max \left\{\frac{d_{2}}{d_{1}}, \frac{1}{\beta}\right\}\right)^{2 k}}{4 \beta} \int_{\Omega} \varphi^{2} d x \\
\leq & \frac{k \sigma c^{k-1}\left(\max \left\{\frac{d_{2}}{d_{1}}, \frac{1}{\beta}\right\}\right)^{k-1}}{\lambda_{1}} \int_{\Omega}|\nabla \phi|^{2} d x \\
& +\frac{c^{2 k} \delta^{2}\left(\max \left\{\frac{d_{2}}{d_{1}}, \frac{1}{\beta}\right\}\right)^{2 k}}{4 \beta \lambda_{1}} \int_{\Omega}|\nabla \varphi|^{2} d x .
\end{aligned}
$$

Similarly, multiplying $\varphi$ to the second equation of (3) and integrating by parts over domain $\Omega$, we have

$$
d_{2} \int_{\Omega}|\nabla \varphi|^{2} d x
$$$$
=\int_{\Omega}\left(c-u^{k} f(v)-v\right) \varphi d x
$$$$
=c \int_{\Omega} \varphi d x-\int_{\Omega} v \varphi d x-\int_{\Omega} u^{k} f(v) \varphi d x
$$$$
=-\int_{\Omega} \varphi^{2} d x-\int_{\Omega}\left(u^{k} f(v)-\bar{u}^{k} f(\bar{v})\right) \varphi d x,
$$$$
\leq-\int_{\Omega} \varphi^{2} d x-k \sigma c^{k-1}\left(\max \left\{\frac{d_{2}}{d_{1}}, \frac{1}{\beta}\right\}\right)^{k-1} \int_{\Omega}|\phi \varphi| d x
$$$$
-c^{k} \delta\left(\max \left\{\frac{d_{2}}{d_{1}}, \frac{1}{\beta}\right\}\right)^{k} \int_{\Omega} \varphi^{2} d x
$$

$\leq-\int_{\Omega} \varphi^{2} d x+k \sigma c^{k-1}\left(\max \left\{\frac{d_{2}}{d_{1}}, \frac{1}{\beta}\right\}\right)^{k-1} \int_{\Omega}|\phi \varphi| d x$

$$
\begin{aligned}
& \leq \frac{k^{2} \sigma^{2} c^{2(k-1)}\left(\max \left\{\frac{d_{2}}{d_{1}}, \frac{1}{\beta}\right\}\right)^{2 k-2}}{4} \int_{\Omega} \phi^{2} d x \\
& \leq \frac{k^{2} \sigma^{2} c^{2(k-1)}\left(\max \left\{\frac{d_{2}}{d_{1}}, \frac{1}{\beta}\right\}\right)^{2 k-2}}{4 \lambda_{1}} \int_{\Omega}|\nabla \phi|^{2} d x .
\end{aligned}
$$

As a result, one deduces

$$
\begin{aligned}
& d_{1} \int_{\Omega}|\nabla \phi|^{2} d x+d_{2} \int_{\Omega}|\nabla \psi|^{2} d x \\
\leq & \frac{k \sigma c^{k-1}\left(\max \left\{\frac{d_{2}}{d_{1}}, \frac{1}{\beta}\right\}\right)^{k-1}}{4 \lambda_{1}} \\
& \times\left[4+k \sigma c^{k-1}\left(\max \left\{\frac{d_{2}}{d_{1}}, \frac{1}{\beta}\right\}\right)^{k-1}\right] \int_{\Omega}|\nabla \phi|^{2} d x \\
& +\frac{c^{2 k} \delta^{2}\left(\max \left\{\frac{d_{2}}{d_{1}}, \frac{1}{\beta}\right\}\right)^{2 k}}{4 \beta \lambda_{1}} \int_{\Omega}|\nabla \varphi|^{2} d x \\
= & d_{1}^{*} \int_{\Omega}|\nabla \phi|^{2} d x+d_{2}^{*} \int_{\Omega}|\nabla \varphi|^{2} d x,
\end{aligned}
$$

where $d_{1}^{*}$ and $d_{2}^{*}$ are set to be

$$
\begin{aligned}
& d_{1}^{*}=\frac{k \sigma c^{k-1}\left(\max \left\{\frac{d_{2}}{d_{1}}, \frac{1}{\beta}\right\}\right)^{k-1}}{4 \lambda_{1}} \\
& \times\left[4+k \sigma c^{k-1}\left(\max \left\{\frac{d_{2}}{d_{1}}, \frac{1}{\beta}\right\}\right)^{k-1}\right], \\
& d_{2}^{*}=\frac{c^{2 k} \delta^{2}\left(\max \left\{\frac{d_{2}}{d_{1}}, \frac{1}{\beta}\right\}\right)^{2 k}}{4 \beta \lambda_{1}} .
\end{aligned}
$$

Thence, if there is a $d^{*}=d^{*}(c, k, \beta, \sigma, \delta, \Omega)$ such that $\min \left\{d_{1}, d_{2}\right\}>\max \left\{d_{1}^{*}, d_{2}^{*}\right\}:=d^{*}$,

holds, then one has $\nabla \phi=\nabla \varphi=0$, this indicates that $(u(x), v(x))$ must be a positive constant solution of elliptic system (3).

Theorem 3 Suppose that $c, \beta, d_{1}, d_{2}, k \geq 2$ are positive constants in (3) and $\Omega \subset R^{n}$ is a bounded domain with sufficient smooth boundary, then elliptic system (3) has no non-constant steady state solution if

$$
\frac{\left(d_{1}+2 \beta d_{2}^{2}\right)\left[k^{2} \sigma^{2} c^{2(k-1)}\left(\max \left\{\frac{d_{2}}{d_{1}}, \frac{1}{\beta}\right\}\right)^{2 k-2}\right]}{8 \beta \lambda_{1}^{2} d_{1}^{2} d_{2}}<1 .
$$

Proof. Consider that multiplying Eq. (7) by $\phi$ and integrating it by parts, we have

$-\int_{\Omega} \nabla \omega \nabla \phi d x=\beta \int_{\Omega} \phi^{2} d x+\int_{\Omega} \phi \varphi d x$.

From Eq. (6), one obtains

$\int_{\Omega} \nabla \omega d x=d_{1} \int_{\Omega} \nabla u d x+d_{2} \int_{\Omega} \nabla v d x$

we therefore obtain

$\int_{\Omega} \nabla \omega \nabla \phi d x=d_{1} \int_{\Omega}|\nabla \phi|^{2} d x+d_{2} \int_{\Omega} \nabla \phi \nabla \varphi d x$. 
Combining Eqs. (8) and (9), we have

$$
\begin{aligned}
d_{2} \int_{\Omega} \nabla \phi \nabla \varphi d x= & -d_{1} \int_{\Omega}|\nabla \phi|^{2} d x-\beta \int_{\Omega} \phi^{2} d x \\
& -\int_{\Omega} \phi \varphi d x .
\end{aligned}
$$

Then, by employing Eqs. (6) and (10), one has

$$
\begin{aligned}
0 \leq \int_{\Omega}|\nabla \omega|^{2} d x= & d_{1}^{2} \int_{\Omega}|\nabla \phi|^{2} d x+2 d_{1} d_{2} \int_{\Omega} \nabla \phi \nabla \varphi d x \\
& +d_{2}^{2} \int_{\Omega}|\nabla \varphi|^{2} d x \\
= & d_{2}^{2} \int_{\Omega}|\nabla \varphi|^{2} d x-d_{1}^{2} \int_{\Omega}|\nabla \phi|^{2} d x \\
& -2 \beta d_{1} \int_{\Omega} \phi^{2} d x-2 d_{1} \int_{\Omega} \phi \varphi d x .
\end{aligned}
$$

Therefore, the Poincaré's inequality and Cauchy-Schwarz show that

$$
\begin{aligned}
& d_{1}^{2} \int_{\Omega}|\nabla \phi|^{2} d x+2 \beta d_{1} \int_{\Omega} \phi^{2} d x \\
\leq & d_{2}^{2} \int_{\Omega}|\nabla \varphi|^{2} d x+2 d_{1} \int_{\Omega}|\phi \varphi| d x \\
\leq & d_{2}^{2} \int_{\Omega}|\nabla \varphi|^{2} d x+2 d_{1} \sqrt{\int_{\Omega} \phi^{2} d x \int_{\Omega} \varphi^{2} d x} \\
\leq & d_{2}^{2} \int_{\Omega}|\nabla \varphi|^{2} d x+2 \beta d_{1} \int_{\Omega} \phi^{2} d x+\frac{d_{1}}{2 \beta} \int_{\Omega} \varphi^{2} d x \\
\leq & d_{2}^{2} \int_{\Omega}|\nabla \varphi|^{2} d x+2 \beta d_{1} \int_{\Omega} \phi^{2} d x+\frac{d_{1}}{2 \beta \lambda_{1}} \int_{\Omega}|\nabla \varphi|^{2} d x .
\end{aligned}
$$

It then leads to

$$
\begin{aligned}
& \int_{\Omega}|\nabla \phi|^{2} d x \\
\leq & \frac{d_{1}+2 \beta d_{2}^{2}}{2 \beta \lambda_{1} d_{1}^{2}} \int_{\Omega}|\nabla \varphi|^{2} d x \\
\leq & \frac{\left(d_{1}+2 \beta d_{2}^{2}\right)\left[k^{2} \sigma^{2} c^{2(k-1)}\left(\max \left\{\frac{d_{2}}{d_{1}}, \frac{1}{\beta}\right\}\right)^{2 k-2}\right]}{8 \beta \lambda_{1}^{2} d_{1}^{2} d_{2}} \\
& \times \int_{\Omega}|\nabla \phi|^{2} d x .
\end{aligned}
$$

Consequently, we claim that $(u(x), v(x))$ must be a constant solution of elliptic system (3) if

$$
\frac{\left(d_{1}+2 \beta d_{2}^{2}\right)\left[k^{2} \sigma^{2} c^{2(k-1)}\left(\max \left\{\frac{d_{2}}{d_{1}}, \frac{1}{\beta}\right\}\right)^{2 k-2}\right]}{8 \beta \lambda_{1}^{2} d_{1}^{2} d_{2}}<1 .
$$

The desired result is confirmed.
Remark 1 By means of Theorem 2.3 we find a fact that

$$
\lim _{d_{1} \rightarrow \infty} \frac{\left(d_{1}+2 \beta d_{2}^{2}\right)\left[k^{2} \sigma^{2} c^{2(k-1)}\left(\max \left\{\frac{d_{2}}{d_{1}}, \frac{1}{\beta}\right\}\right)^{2 k-2}\right]}{8 \beta \lambda_{1}^{2} d_{1}^{2} d_{2}}
$$$$
=0<1 \text {. }
$$

This indicates that system (3) always has no non-constant solution if $d_{1}$ is large enough and $d_{2}$ is fixed. Thence, in view of the spatial or spatiotemporal patterns of system (3) we should restrict $d_{1}<d_{2}$, namely, the diffusion rate of activator should great than the diffusion rate of substrate. Otherwise, there might be no pattern formation of system (3).

\section{Spatiotemporal patterns in the networked model}

In this section, we shall investigate the Hopf bifurcation, Turing instability and the Turing-Hopf bifurcation of the networked system (2). It is easy to check out that system (2) has a positive equilibrium $E_{*}=\left(u_{*}, v_{*}\right)=$ $\left(u_{*}, c-\beta u_{*}\right)$ when $0<u_{*}<\frac{c}{\beta}$. Keeping this in mind, we now perform the linear stability analysis of system (2) near internal positive equilibria $E_{*}=\left(u_{*}, v_{*}\right)$. To do so, let $\delta u_{i}=u_{i}-u_{*}$ and $\delta v_{i}=v_{i}-v_{*}$, then the linear system of (2) evaluated at $E_{*}=\left(u_{*}, v_{*}\right)$ can be described as follows

$\left(\begin{array}{c}\delta \dot{u}_{i} \\ \delta \dot{v}_{i}\end{array}\right)=J\left(\begin{array}{c}\delta u_{i} \\ \delta v_{i}\end{array}\right)+D\left(\begin{array}{c}\sum_{j=1}^{N} \Delta_{i j} \delta u_{j} \\ \sum_{j=1}^{N} \Delta_{i j} \delta v_{j}\end{array}\right)$,

where

$J=\left(\begin{array}{cc}F_{u} & F_{v} \\ G_{u} & G_{v}\end{array}\right), \quad D=\left(\begin{array}{cc}d_{1} & 0 \\ 0 & d_{2}\end{array}\right)$,

with

$$
\begin{gathered}
F_{u}=(k-1) \beta>0, F_{v}=u_{*}^{k} f^{\prime}\left(v_{*}\right)>0, \\
G_{u}=-k \beta<0, G_{v}=-u_{*}^{k} f^{\prime}\left(v_{*}\right)-1<0 .
\end{gathered}
$$

Let $0=\Lambda_{1}>\Lambda_{2}>\cdots>\Lambda_{N}$ be the eigenvalues of the discrete Laplacian matrix $\boldsymbol{\Delta}$, and we assume that $L_{\phi}=\left\{\phi_{i}\right\}_{i=1}^{N}$ is the subspace generated by the eigenfunctions associated to the topological eigenvalue $\Lambda_{i}$. As a result, the general solution of system (2) can be written as follows

$$
\left(\begin{array}{c}
\delta u_{i} \\
\delta v_{i}
\end{array}\right)=\sum_{i=1}^{N}\left(\begin{array}{c}
a_{i} \\
b_{i}
\end{array}\right) \phi_{i} e^{\lambda_{i} t},
$$


with $\sum_{j=1}^{N} \Delta_{i j} \phi_{j}=\Lambda_{i} \phi_{i}$. Putting (12) into (11), then for each node $i=1,2, \cdots N$, we have

$\lambda_{i}\left(\begin{array}{c}a_{i} \\ b_{i}\end{array}\right) \phi_{i} e^{\lambda_{i} t}=J\left(\begin{array}{c}a_{i} \\ b_{i}\end{array}\right) \phi_{i} e^{\lambda_{i} t}+\Lambda_{i} D\left(\begin{array}{c}a_{i} \\ b_{i}\end{array}\right) \phi_{i} e^{\lambda_{i} t}$.

It follows that

$\left|\lambda_{i} I-J-\Lambda_{i} D\right|=0$, for $i=1,2, \cdots N$.

Hence, we have the characteristic equation for each node with the form

$\lambda_{i}^{2}-T_{\Lambda_{i}}\left(d_{2}, \beta\right) \lambda_{i}+D_{\Lambda_{i}}\left(d_{2}, \beta\right)=0$, for $i=1,2, \cdots N$,

where

$$
\left\{\begin{aligned}
T_{\Lambda_{i}}\left(d_{2}, \beta\right)= & \left(d_{1}+d_{2}\right) \Lambda_{i}+F_{u}+G_{v} \\
D_{\Lambda_{i}}\left(d_{2}, \beta\right)= & d_{1} d_{2} \Lambda_{i}^{2}+\left(d_{1} G_{v}+d_{2} F_{u}\right) \Lambda_{i} \\
& +F_{u} G_{v}-G_{u} F_{v}
\end{aligned}\right.
$$

with $F_{u}+G_{v}=(k-1) \beta-u_{*}^{k} f^{\prime}\left(v_{*}\right)-1$ and $F_{u} G_{v}-$ $G_{u} F_{v}=\beta\left(u_{*}^{k} f^{\prime}\left(v_{*}\right)-k+1\right)$. Then the characteristic roots of Eq. (13) can be exhibited of the form

$\lambda_{i}=\frac{T_{\Lambda_{i}}\left(d_{2}, \beta\right) \pm \sqrt{T_{\Lambda_{i}}^{2}\left(d_{2}, \beta\right)-4 D_{\Lambda_{i}}\left(d_{2}, \beta\right)}}{2}$.

Now, we have stability results of the internal positive equilibrium $E_{*}$ as follows.

Theorem 4 Suppose that $c, \beta, k \geq 2$ are positive constants, $0<u_{*}<\frac{c}{\beta}$ and $d_{1}=d_{2}=0$. Then

(i) if $\beta_{H}<\frac{k}{k-1}$, then $E_{*}=\left(u_{*}, v_{*}\right)$ is unstable;

(ii) if $0<\frac{k}{k-1}<\beta_{H}$ and $\beta>\beta_{H}$, then $E_{*}=\left(u_{*}, v_{*}\right)$ is unstable;

(iii) if $0<\frac{k}{k-1}<\beta_{H}$ and $\beta<\beta_{H}$, then $E_{*}=\left(u_{*}, v_{*}\right)$ is locally asymptotically stable;

(iv) if $0<\frac{k}{k-1}<\beta_{H}$ and $\beta=\beta_{H}$, then networked system (2) undergoes a Hopf bifurcation, where $\beta_{H}=$ $\frac{u_{*}^{k} f^{\prime}\left(v_{*}\right)+1}{k-1}$.

Proof. Recall that Eqs. (13) and (15), the stability of the positive equilibrium $E_{*}=\left(u_{*}, v_{*}\right)$ can be determined by the sign of $T_{\Lambda_{i}}\left(d_{2}, \beta\right)$ and $D_{\Lambda_{i}}\left(d_{2}, \beta\right)$. That is the positive equilibrium $E_{*}=\left(u_{*}, v_{*}\right)$ is locally asymptotically stable if $T_{\Lambda_{i}}\left(d_{2}, \beta\right)<0$ and $D_{\Lambda_{i}}\left(d_{2}, \beta\right)>0$. Here if $d_{1}=d_{2}=0$, then we denote $T_{0}\left(d_{2}, \beta\right)=F_{u}+$ $G_{v}=(k-1) \beta-u_{*}^{k} f^{\prime}\left(v_{*}\right)-1$ and $D_{0}\left(d_{2}, \beta\right)=F_{u} G_{v}-$ $G_{u} F_{v}=\beta\left(u_{*}^{k} f^{\prime}\left(v_{*}\right)-k+1\right)$. A straightforward computation shows that $D_{0}\left(d_{2}, \beta\right)<0$ if $\beta_{H}<\frac{k}{k-1}$. This implies there is at least one eigenvalue of the characteristic equation (13) with positive real part, so $E_{*}=\left(u_{*}, v_{*}\right)$ is unstable, no matter what the sign of $T_{0}\left(d_{2}, \beta\right)$ is. Conclusion (i) is valid. Condition $0<\frac{k}{k-1}<\beta_{H}$ indicates $D_{0}\left(d_{2}, \beta\right)>0$. Hence, it is easy to verify (ii) and (iii) are true. On the other hand, taking the derivatives with respect to $\beta$ yield

$\left.\frac{\mathrm{dRe} \lambda}{\mathrm{d} \beta}\right|_{\beta=\beta_{H}}=\frac{k-1}{2}>0$.

Thence, the Poincaré-Andronov-Hopf bifurcation theorem shows that the local system of (2) undergoes the Hopf bifurcation at $E_{*}=\left(u_{*}, v_{*}\right)$ when $\beta=\beta_{H}$. This completes the proof.

In what follows, we focus on the Turing instability of the positive equilibrium $E_{*}=\left(u_{*}, v_{*}\right)$ when diffusion is presented.

Theorem 5 Suppose that $c, \beta, k \geq 2$ are positive constants, $0<u_{*}<\frac{c}{\beta}, 0<\frac{k}{k-1}<\beta_{H}$ and $\beta<\beta_{H}$. Then

(i) if $\frac{F_{u}}{d_{1}} \leq 1$, then $E_{*}=\left(u_{*}, v_{*}\right)$ is locally asymptotically stable;

(ii) if $\frac{F_{u}}{d_{1}}>\left(-\Lambda_{i c}\right)$ and $d_{2}<d_{2}^{T}$, then $E_{*}=\left(u_{*}, v_{*}\right)$ is locally asymptotically stable;

(iii) if $\frac{F_{u}}{d_{1}}>\left(-\Lambda_{i c}\right)$ and $d_{2}>d_{2}^{T}$, then $E_{*}=\left(u_{*}, v_{*}\right)$ is unstable, it implies that the networked system (2) undergoes a Turing instability, where

$d_{2}^{T}=\frac{d_{1} G_{v}\left(-\Lambda_{i c}\right)-D_{0}}{\left(-d_{1} \Lambda_{i c}-F_{u}\right)\left(-\Lambda_{i c}\right)}>0$,

for some suitable $\Lambda_{i c} \in\left\{\Lambda_{1}, \Lambda_{2} \cdots \Lambda_{N}\right\}$.

Proof. Form Theorem 4, we know that $E_{*}=\left(u_{*}, v_{*}\right)$ is locally asymptotically stable when $0<\frac{k}{k-1}<\beta_{H}$ and $\beta<\beta_{H}$. This leads to $T_{0}\left(d_{2}, \beta\right)<0, D_{0}\left(d_{2}, \beta\right)>0$. On the other hand, notice that $0=\Lambda_{1}>\Lambda_{2}>\cdots>\Lambda_{N}$, then we have $T_{\Lambda_{i}}\left(d_{2}, \beta\right)=\left(d_{1}+d_{2}\right) \Lambda_{i}+T_{0}<0$. Hence, the stability of positive equilibrium $E_{*}$ is determined by sign of $D_{\Lambda_{i}}\left(d_{2}, \beta\right)$. Now, rewriting $D_{\Lambda_{i}}\left(d_{2}, \beta\right)$ in the following form

$$
\begin{aligned}
& D_{\Lambda_{i}}\left(d_{2}, \beta\right) \\
= & d_{1} d_{2} \Lambda_{i}^{2}+\left(d_{1} G_{v}+d_{2} F_{u}\right) \Lambda_{i}+F_{u} G_{v}-G_{u} F_{v}, \\
= & d_{1} d_{2}\left(-\Lambda_{i}\right)^{2}-\left(d_{1} G_{v}+d_{2} F_{u}\right)\left(-\Lambda_{i}\right)+D_{0} \\
= & d_{1} d_{2}\left[\left(-\Lambda_{i}\right)-\frac{F_{u}}{d_{1}}\right]\left(-\Lambda_{i}\right)+D_{0}-d_{1} G_{v}\left(-\Lambda_{i}\right) \\
= & \left.d_{2}\left(d_{1}\left(-\Lambda_{i}\right)-F_{u}\right)\right)\left(-\Lambda_{i}\right)+D_{0}-d_{1} G_{v}\left(-\Lambda_{i}\right) .
\end{aligned}
$$

It is noticed that $F_{u}>0$ and $G_{v}<0$, we thus obtain $D_{0}-d_{1} G_{v}\left(-\Lambda_{i}\right)>0$ for all $i=1,2, \cdots N$. Hence, if $\frac{F_{u}}{d_{1}} \leq$ 1 then we have $-\frac{F_{u}}{d_{1}} \geq-1 \geq \Lambda_{i}$, namely $\left(-\Lambda_{i}\right)-\frac{F_{u}}{d_{1}} \geq$ 0 . It indicates that $D_{\Lambda_{i}}\left(d_{2}, \beta\right)>0$ for all $i=1,2, \cdots N$. Then all eigenvalues of the characteristic equation (13) have negative real parts, namely, $E_{*}=\left(u_{*}, v_{*}\right)$ is locally asymptotically stable.

If $\frac{F_{u}}{d_{1}}>\left(-\Lambda_{i c}\right)$, then one has $d_{1}\left(-\Lambda_{i c}\right)-F_{u}<0$. This means $d_{2}^{T}>0$ for some suitable $\Lambda_{i c} \in\left\{\Lambda_{1}, \Lambda_{2} \cdots\right.$ $\left.\Lambda_{N}\right\}$. On the other hand, if $d_{2}<d_{2}^{T}$, one obtains

$d_{2}<d_{2}^{T} \leq \frac{d_{1} G_{v}\left(-\Lambda_{i c}\right)-D_{0}}{\left(-d_{1} \Lambda_{i c}-F_{u}\right)\left(-\Lambda_{i c}\right)}$, 
this gives $\left.D_{\Lambda_{i c}}\left(d_{2}, \beta\right)=d_{2}\left(d_{1}\left(-\Lambda_{i c}\right)-F_{u}\right)\right)\left(-\Lambda_{i c}\right)+$ $D_{0}-d_{1} G_{v}\left(-\Lambda_{i c}\right)>0$. Consequently, all eigenvalues of the characteristic equation (13) have negative real parts, namely $E_{*}=\left(u_{*}, v_{*}\right)$ is locally asymptotically stable. (ii) is true. By the same manner, one could obtain $D_{\Lambda_{i c}}\left(d_{2}, \beta\right)<0$ if $\frac{F_{u}}{d_{1}}>\left(-\Lambda_{i c}\right)$ and $d_{2}>d_{2}^{T}$. This indicates there is at least one eigenvalue of the characteristic equation (13) has positive real part. As a result, the networked system (2) may admit a Turing instability. The proof is finished.

We now proceed to exhibit a result of the TuringHopf bifurcation as below.

Theorem 6 Suppose that $c, \beta, k \geq 2$ are positive constants, $0<u_{*}<\frac{c}{\beta}$. Then the networked system (2) undergoes the Turing-Hopf bifurcation at $\left(d_{2}, \beta\right)=\left(d_{2}^{*}, \beta^{*}\right)$, where

$$
\left\{\begin{array}{l}
\beta^{*}=\frac{u_{*}^{k} f^{\prime}\left(v_{*}\right)+1}{k-1}, \\
d_{2}^{*}=\frac{\left(u_{*}^{k} f^{\prime}\left(v_{*}\right)+1\right)\left[d_{1}(k-1)\left(-\Lambda_{i}^{c}\right)+u_{*}^{k} f^{\prime}\left(v_{*}\right)-k+1\right]}{(1-k)\left(-d_{1} \Lambda_{i}^{c}-u_{*}^{k} f^{\prime}\left(v_{*}\right)-1\right)\left(-\Lambda_{i}^{c}\right)},
\end{array}\right.
$$

if there exists some suitable $\Lambda_{i}^{c} \in\left\{\Lambda_{1}, \Lambda_{2} \cdots \Lambda_{N}\right\}$.

Proof. For the purpose of obtaining the TuringHopf bifurcation point $\left(d_{2}, \beta\right)=\left(d_{2}^{*}, \beta^{*}\right)$, one should guarantee the Hopf bifurcation curve and the Turing bifurcation curve intersect. By Theorem 4 and Theorem 5 , we know that the networked system (2) admits the Hopf bifurcation at $\beta=\beta_{H}$ and the Turing bifurcation when $d_{2}=d_{2}^{T}$. Hence, for the Turing-Hopf bifurcation, the following equations should be satisfied, that is

$$
\left\{\begin{array}{l}
T_{0}\left(d_{2}, \beta\right)=0 \\
D_{\Lambda_{i}}\left(d_{2}, \beta\right)=0
\end{array}\right.
$$

Consequently, we have

$$
\left\{\begin{array}{l}
\beta^{*}=\frac{u_{*}^{k} f^{\prime}\left(v_{*}\right)+1}{k-1}, \\
d_{2}^{*}=\frac{\left(u_{*}^{k} f^{\prime}\left(v_{*}\right)+1\right)\left[d_{1}(k-1)\left(-\Lambda_{i}^{c}\right)+u_{*}^{k} f^{\prime}\left(v_{*}\right)-k+1\right]}{(1-k)\left(-d_{1} \Lambda_{i}^{c}-u_{*}^{k} f^{\prime}\left(v_{*}\right)-1\right)\left(-\Lambda_{i}^{c}\right)} .
\end{array}\right.
$$

Also, a simple calculation shows that

$$
\left.\frac{\mathrm{dRe} \lambda}{\mathrm{d} d_{2}}\right|_{d_{2}=d_{2}^{*}}=\frac{\Lambda_{i}^{c}\left(d_{1} \Lambda_{i}^{c}+F_{u}\right)}{T_{\Lambda_{i}^{c}}\left(d_{2}^{*}, \beta\right)} \neq 0
$$

for some suitable $\Lambda_{i}^{c} \in\left\{\Lambda_{1}, \Lambda_{2} \cdots \Lambda_{N}\right\}$. This combine with Eq. (16) we claim that the results in Theorem 6 are valid.

\section{An application}

In this section, we shall verify the results exhibited in Theorem 4-6. To do so, we set $k=2$ and $f(v)=v$, then the network system (2) becomes:

$$
\left\{\begin{array}{l}
\frac{d u_{i}}{d t}-d_{1} \sum_{j=1}^{N} \Delta_{i j} u_{i}=u_{i}^{2} v_{i}-\beta u_{i}, \\
\frac{d v_{i}}{d t}-d_{2} \sum_{j=1}^{N} \Delta_{i j} v_{i}=c-u_{i}^{2} v_{i}-v_{i} .
\end{array}\right.
$$

Let $\alpha=\frac{c}{\beta}$. Then when $\alpha=2$, the system (18) has a unique internal positive equilibrium $E_{1}=(1, \beta)$, and when $\alpha>2$, system (18) has two internal positive equilibrium $E_{2}$ and $E_{3}$, where

$$
\begin{aligned}
& E_{2}=\left(\frac{\alpha-\sqrt{\alpha^{2}-4}}{2}, \frac{\alpha+\sqrt{\alpha^{2}-4}}{2} \beta\right), \\
& E_{3}=\left(\frac{\alpha+\sqrt{\alpha^{2}-4}}{2}, \frac{\alpha-\sqrt{\alpha^{2}-4}}{2} \beta\right) .
\end{aligned}
$$

By [23], the internal positive equilibrium $E_{1}$ is nonhyperbolic, and $E_{2}$ is a unstable saddle. Therefore, we shall main focus on the dynamical behaviors of the internal positive equilibrium $E_{3}$, and we denote it by $E_{*}$, that is $E_{3}=E_{*}=\left(u_{*}, v_{*}\right)$, where

$u_{*}=\frac{\alpha+\sqrt{\alpha^{2}-4}}{2}, \quad v_{*}=\frac{\alpha-\sqrt{\alpha^{2}-4}}{2} \beta$.

Obviously, we have $0<u_{*}<\alpha$. Then we obtain the fact that

$F_{u}=\beta>0, \quad F_{v}=\frac{\alpha^{2}-2+\alpha \sqrt{\alpha^{2}-4}}{2}>0$,

$G_{u}=-2 \beta<0, \quad G_{v}=-\frac{\alpha^{2}+\alpha \sqrt{\alpha^{2}-4}}{2}<0$.

As a result, we exhibit the main results of system (18) as follows:

Theorem 7 Suppose that $c, \beta$ are positive constants, $\alpha>2$ and $d_{1}=d_{2}=0$. Then

(i) if $\beta_{H}<2$, then $E_{*}=\left(u_{*}, v_{*}\right)$ is unstable;

(ii) if $0<2<\beta_{H}$ and $\beta>\beta_{H}$, then $E_{*}=\left(u_{*}, v_{*}\right)$ is unstable;

(iii) if $0<2<\beta_{H}$ and $\beta<\beta_{H}$, then $E_{*}=\left(u_{*}, v_{*}\right)$ is locally asymptotically stable;

(iv) if $0<2<\beta_{H}$ and $\beta=\beta_{H}$, then network system (2) undergoes a Hopf bifurcation, where $\beta_{H}=\frac{\alpha\left(\alpha+\sqrt{\alpha^{2}-4}\right)}{2}$.

Proof. It is easy to obtain

$$
\left\{\begin{array}{l}
T_{0}\left(d_{2}, \beta\right)=F_{u}+G_{v}=\beta-\frac{\alpha^{2}+\alpha \sqrt{\alpha^{2}-4}}{2}, \\
D_{0}\left(d_{2}, \beta\right)=F_{u} G_{v}-G_{u} F_{v}=\frac{\alpha^{2}-4+\alpha \sqrt{\alpha^{2}-4}}{2} \beta .
\end{array}\right.
$$

Then, based on Theorem 4 the results in Theorem 7 are valid.

Theorem 8 Suppose that $c, \beta$ are positive constants, $\alpha>2,0<2<\beta_{H}$ and $\beta<\beta_{H}$. Then

(i) if $\frac{\beta}{d_{1}} \leq 1$, then $E_{*}=\left(u_{*}, v_{*}\right)$ is locally asymptotically stable; 
(ii) if $\frac{\beta}{d_{1}}>\left(-\Lambda_{i c}\right)$ and $d_{2}<d_{2}^{T}$, then $E_{*}=\left(u_{*}, v_{*}\right)$ is locally asymptotically stable;

(iii) if $\frac{\beta}{d_{1}}>\left(-\Lambda_{i c}\right)$ and $d_{2}>d_{2}^{T}$, then $E_{*}=\left(u_{*}, v_{*}\right)$ is unstable, it implies that the network system (2) undergoes a Turing instability, where

$d_{2}^{T}=\frac{d_{1} G_{v}\left(-\Lambda_{i c}\right)-D_{0}\left(d_{2}, \beta\right)}{\left(-d_{1} \Lambda_{i c}-\beta\right)\left(-\Lambda_{i c}\right)}>0$,

for some suitable $\Lambda_{i c} \in\left\{\Lambda_{1}, \Lambda_{2} \cdots \Lambda_{N}\right\}$, in which $G_{v}$ and $D_{0}\left(d_{2}, \beta\right)$ can be found as before.

Proof. Similar to the proof of Theorem 5, here we omit the detailed proof process.

Theorem 9 Suppose that $c, \beta$ are positive constants and $\alpha>2$. Then the network system (2) undergoes the Turing-Hopf bifurcation at $\left(d_{2}, \beta\right)=\left(d_{2}^{*}, \beta^{*}\right)$, where

$$
\left\{\begin{array}{l}
\beta^{*}=\frac{\alpha\left(\alpha+\sqrt{\alpha^{2}-4}\right)}{2}, \\
d_{2}^{*}=\frac{\left(u_{*}^{2}+1\right)\left[d_{1}\left(-\Lambda_{i}^{c}\right)+u_{*}^{2}-1\right]}{\left(d_{1} \Lambda_{i}^{c}+u_{*}^{2}+1\right)\left(-\Lambda_{i}^{c}\right)},
\end{array}\right.
$$

for some suitable $\Lambda_{i}^{c} \in\left\{\Lambda_{1}, \Lambda_{2} \cdots \Lambda_{N}\right\}$.

Proof. By the same manner in Theorem 6, one could show these results, here we omit the detailed proof process.

\section{Numerical simulations}

In Sect. 4, we perform the stability analysis and bifurcations analysis of the networked system (18), and the related results have been exhibited in the Theorems 7-9. Hence, we shall carry out some numerical simulations to verify these results. We fix $N=100$, that is the numbers of the nodes in the random network system (2) are 100. Moreover, we set $p(0 \leq p \leq 1)$ is the connection probability of different nodes $u_{i}$ and $u_{j}$. Firstly,
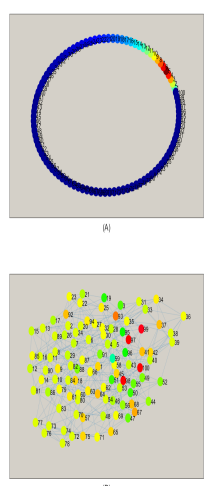
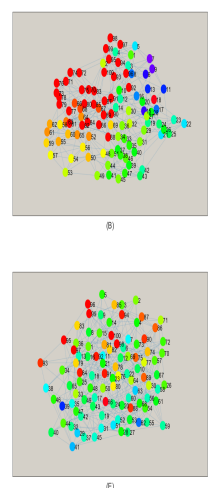
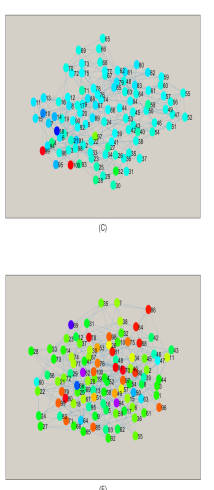

Fig. 1 The distribution of $u_{i}$ in the Watts-Strogatz random network at moment $t=100$. The connection probability $p$ for (A)-(F) are $0,0.1,0.2,0.3,0.4$ and 0.5 , respectively. Here parameters are $c=2.2, \beta=1, d_{1}=1.5$ and $d_{2}=10$. to exhibit the distribution of the solutions $u_{i}$ and $v_{i}$ of system (2), here we assume that the network is a WattsStrogatz type [29]. From the point view of mathematics, a Watts-Strogatz network is an undirected graph, and some of the connections between the nodes are determined, and some of them are random. It possesses the homogeneous degree distribution [14]. It is noticed that the distribution of solutions $u_{i}$ and $v_{i}$ under WattsStrogatz network is very similar, so we only perform the distribution of $u_{i}$ under Watts-Strogatz network. Here we choose parameters in random network system (2) are $c=2.2, \beta=1, d_{1}=1.5$ and $d_{2}=10$, average degree is 4 , and the connection probability $p$ is variable. It is found that $u_{i}$ and $u_{j}$ will eventually become a closed circle when connection probability $p=0$, where $1 \leq i, j \leq 100$ and $i \neq j$. However, when one choose $0<p<1$ such closed circle will be destroyed. More precisely, $u_{i}$ will randomly walk in different positions at the same time $t$. Hence, connection probability $p$ has a vital influence on the distribution of $u_{i}$, see Fig. 1 for more details.
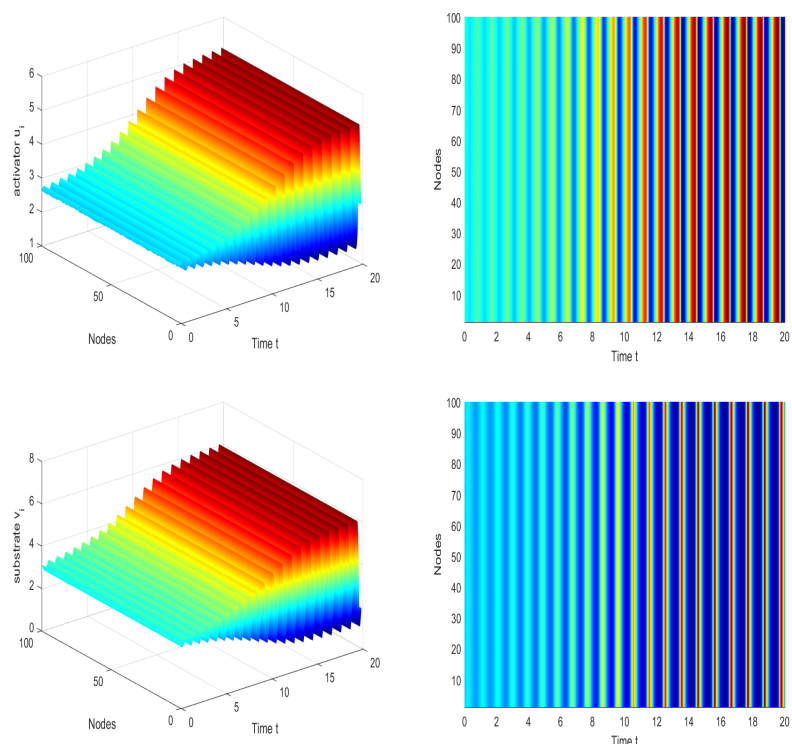

Fig. 2 The random network system (2) undergoes a Hopf bifurcation at positive equilibrium $E_{*}=(2.6180,3.0023)$. Here parameters are $c=23.58, \beta=7.86$ and $p=0.05$.

Now we choose $c=23.58, \beta=7.86$ and connection probability $p=0.05$ then one has $\alpha=3, \beta_{H}=$ $7.8551 \approx 7.86$. Hence, the random networked system (2) undergoes a Hopf bifurcation at positive equilibrium $E_{*}=(2.6180,3.0023)$, and the periodic solution bifurcated from the Hopf bifurcation is spatially homogeneous, see Fig. 2. 

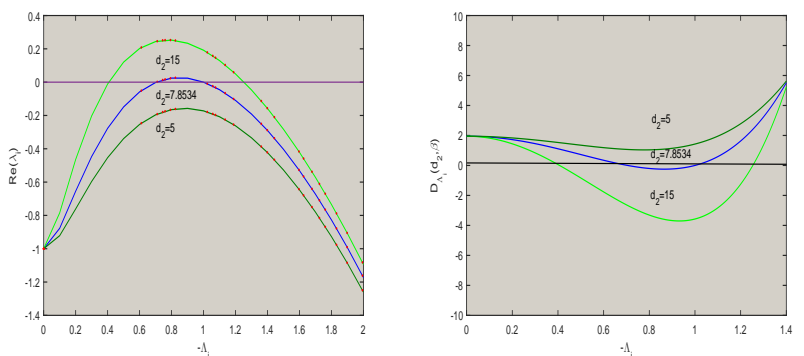

Fig. 3 Left: plot $\operatorname{Re}\left(\lambda_{i}\right)$ against different values of $d_{2}$, and here $(\cdot)$ represents eigenvalue of the discrete Laplacian matrix. Right: plot of $D_{\Lambda_{i}}\left(d_{2}, \beta\right)$ against $-\Lambda_{i}$ for different values of the parameter $d_{2}$. Here parameters are $c=2.3, \beta=1, d_{1}=0.5$ and $p=0.02$.

Figure 3 shows the real parts of the characteristic roots $R e\left(\lambda_{i}\right)$ against different values of $d_{2}$, and $D_{\Lambda_{i}}\left(d_{2}, \beta\right)$ against $-\Lambda_{i}$ for different values of the parameter $d_{2}$, respectively. Here we choose $c=2.3, \beta=1, d_{1}=0.5$ and $p=0.02$, then we know that the critical value of the Turing instability is $d_{2}=d_{2}^{T}=7.8534$ with critical value $\Lambda_{i}=\Lambda_{i c}=-1$. It is found that when $d_{2}>d_{2}^{T}$, then we have $\operatorname{Re}\left(\lambda_{i}\right)>0$ and $D_{\Lambda_{i}}\left(d_{2}, \beta\right)<0$. Hence, the random networked system (2) admits the Turing instability. Also, if $d_{2}<d_{2}^{T}$, then we have $\operatorname{Re}\left(\lambda_{i}\right)<0$ and $D_{\Lambda_{i}}\left(d_{2}, \beta\right)>0$. That means there is no Turing pattern will emerge in the random network system (2).
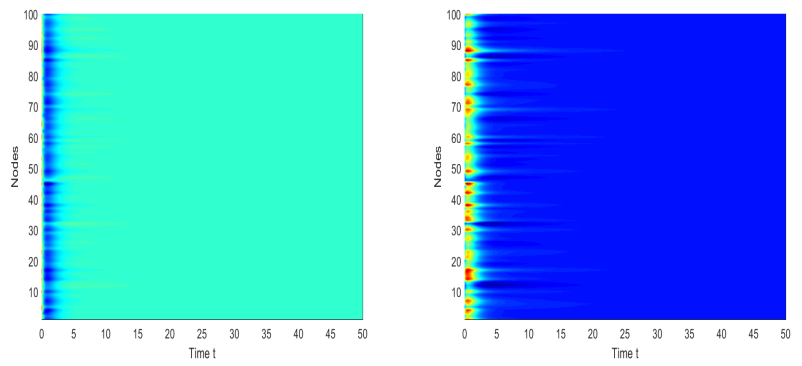

Fig. 4 There is no Turing instability of the random network system (2). Here parameters are $c=2.3, \beta=1, d_{1}=0.5, d_{2}=$ 5 and $p=0.02$.

Figure 4 shows the internal positive equilibrium $E_{*}$ is locally asymptotically stable. Here we choose $d_{2}<$ $d_{2}^{T}$, and the other parameters are fixed in Fig. 3. However, when taking diffusion coefficient $d_{2}=10>d_{2}^{T}$, we find that the Turing instability emerges, and such instability exhibits the stripe patterns, see Fig. 5. Therefore, the theoretical analysis in Theorem 8 are well verified by the numerical simulation results exhibited in Figs. 4 and 5 .

Figure 6 performs the bifurcation diagram in the plane of $\beta-d_{2}$. It is found that the plane of $\beta-d_{2}$
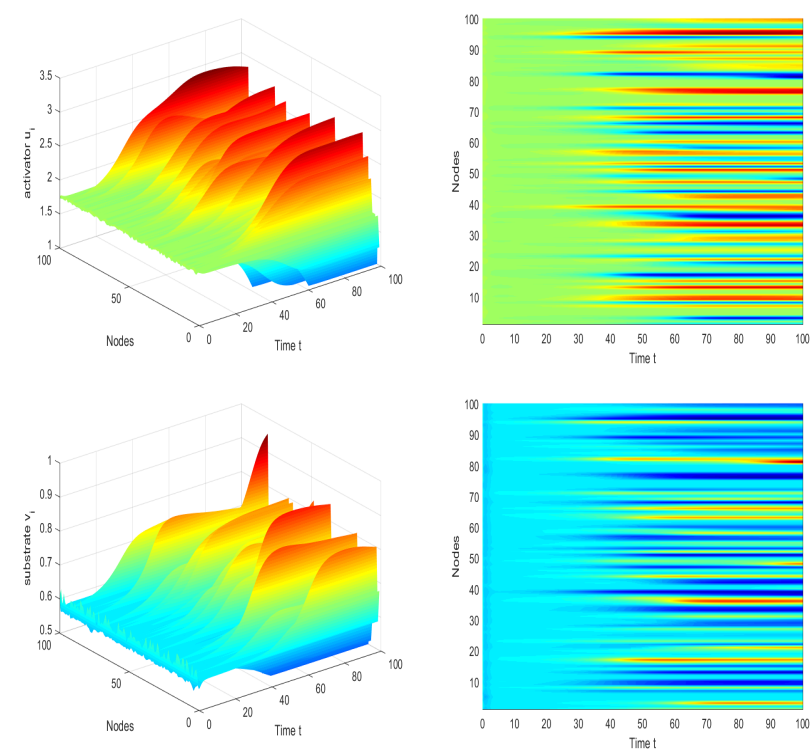

Fig. 5 The random network system (2) admits the Turing instability. Here parameters are $c=2.3, \beta=1, d_{1}=0.5, d_{2}=$ 10 and $p=0.02$.

is divided into four regions, namely stability region, the Hopf instability region, the Turing instability region and the Turing-Hopf bifurcation region, respectively. Obviously, the parameters in Figs. 4 and 5 are located in stale region and the Turing instability region of Fig. 6, respectively.

Now, we choose $c=23.58, d_{1}=2$ and $p=0.02$, then we obtain the fact that $d_{2}=d_{2}^{*}=10.56$ and $\beta=\beta^{*}=7.85$ with critical value $\Lambda_{i}=\Lambda_{i c}=-1$. This implies that the random networked system (2) may undergo a codimensional-two Turing-Hopf bifurcation near the critical point $\left(\beta, d_{2}\right)=(7.85,10.56)$. To do so, we take parameters in the Turing-Hopf region, i.e., $d_{2}=10.6$ and $\beta=7.86$. As a result, the random networked system (2) admits the Turing-Hopf bifurcation, and the spatially inhomogeneous periodic solution can be induced by such codimensional-two bifurcation, see Fig. 7.

In short, all the theoretical analysis in Theorems 7-9 are valid.

To further understand the role of connection probability $p$ in the Turing patterns formation. In what follows, we shall fix the parameters in Fig. 5. That is $c=2.3, \beta=1, d_{1}=0.5, d_{2}=10$ but change the connection probability $p$. We only perform the patterns of $u_{i}$ for convenience.

In Fig. 8, we take the connection probability $p=0$. The numerical simulation result shows that there is no any eigenvalues $-\Lambda_{i}$ of the discrete matrix locate in the Turing instability region. Thence, the internal positive equilibrium $E_{*}$ is asymptotically stable, namely 


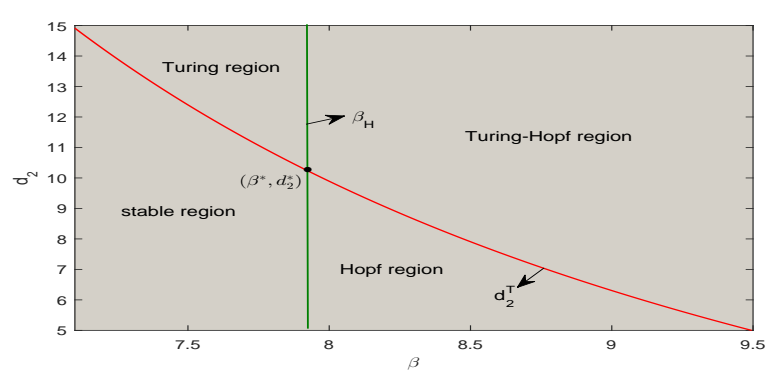

Fig. 6 Bifurcation diagram in the plane of $\beta-d_{2}$. Here $c=$ $23.58, d_{1}=2$, and $\left(\beta^{*}, d_{2}^{*}\right)=(7.85,10.56)$ is the Turing-Hopf bifurcation point.
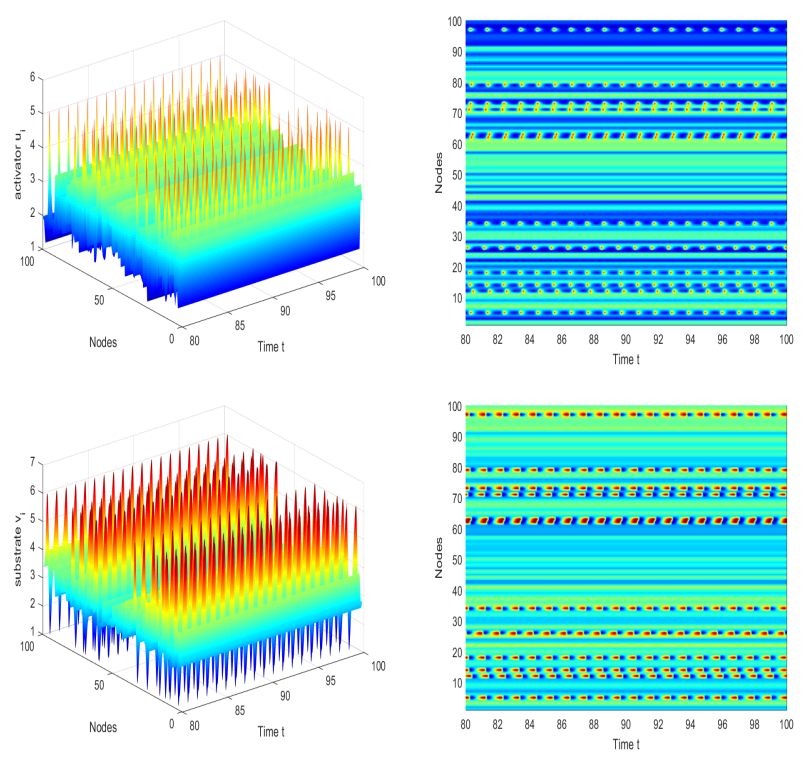

Fig. 7 Spatiotemporal patterns in the random network system (2) induced by the Turing-Hopf bifurcation. Here parameters are $c=23.58, \beta=7.86, d_{1}=2, d_{2}=10.6$ and $p=0.02$.
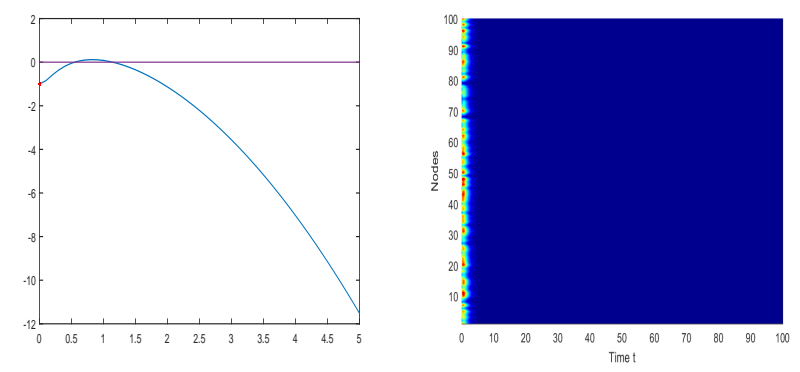

Fig. 8 There is no eigenvalues of the discrete Laplacian matrix locate in Turing instability region, and positive equilibrium $E_{*}$ is stable. Here parameters are $c=2.3, \beta=1, d_{1}=$ $0.5, d_{2}=10$ and $p=0$.

the random networked system (2) will not exhibit the Turing instability.

In Fig. 9, we shall choose the connection probability $p=0.02$. The numerical simulation shows that there are some $-\Lambda_{i}$ in the Turing instability region, i.e., there exists some $-\Lambda_{i}$ in the Turing instability region such that the real parts of characteristic roots $\operatorname{Re}\left(\lambda_{i}\right)>0$. Therefore, the random networked system (2) undergoes a Turing instability.
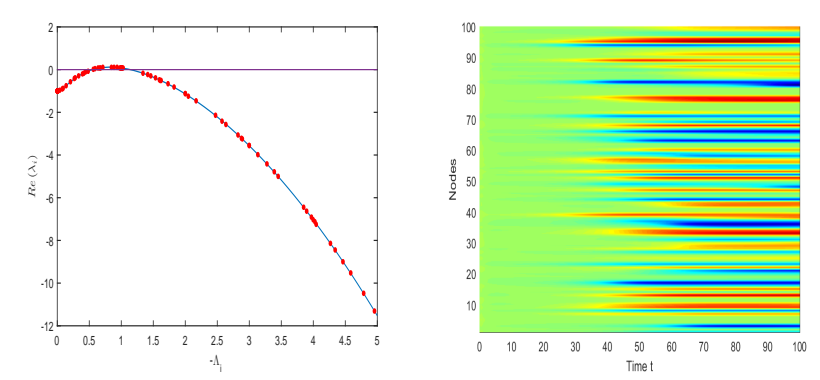

Fig. 9 The Turing instability emerges when there are some eigenvalues of the discrete Laplacian matrix locate in Turing instability region. Here parameters are $c=2.3, \beta=1, d_{1}=$ $0.5, d_{2}=10$ and $p=0.02$.

In Fig. 10, we shall choose the connection probability $p=0.05$. The numerical simulation shows that there are some $-\Lambda_{i}$ in the Turing instability region. However, compared with Fig. 9 there are fewer $-\Lambda_{i}$ in the Turing instability region. Consequently, by comparison we find that there are fewer stripe in the picture.

We then choose the connection probability $p=0.07$ in the Fig. 11. It is found that there are fewer $-\Lambda_{i}$ locate in the Turing instability region than Figs. 9 and 10. More precisely, there is one $-\Lambda_{i}$ lies in the Turing instability region. Hence, the stripe patterns in the picture are fewer. That implies that the Turing instability will eventually happen in the random networked system (2).
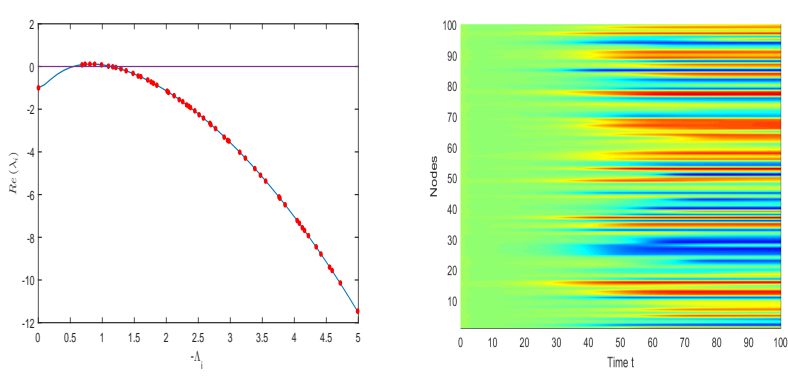

Fig. 10 The Turing instability emerges when there are some eigenvalues of the discrete Laplacian matrix locate in Turing instability region. Here parameters are $c=2.3, \beta=1, d_{1}=$ $0.5, d_{2}=10$ and $p=0.05$.

In Fig. 12, we take the connection probability $p=$ 0.09 , then we find that there is no any $-\Lambda_{i}$ locates in the Turing instability region. That is to say, although 
the same parameters are fixed in the Fig. 5, the Turing pattern will not appear in the random network system (2). And, the right picture of Fig. 12 well verify that such prediction. It is found that the internal positive equilibrium $E_{*}$ becomes a stable one again.
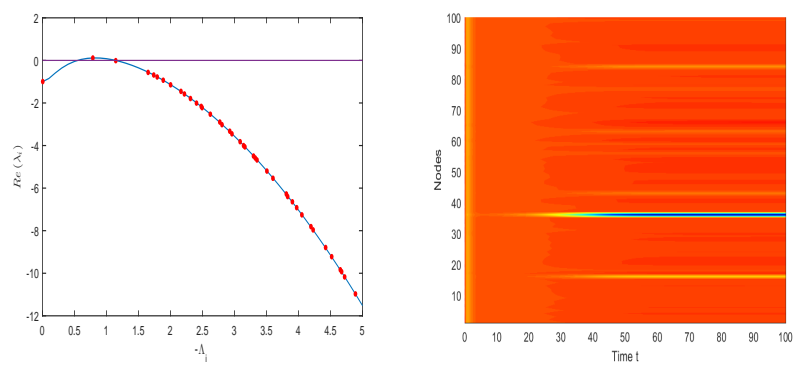

Fig. 11 The Turing instability emerges when there are some eigenvalues of the discrete Laplacian matrix locate in Turing instability region. Here parameters are $c=2.3, \beta=1, d_{1}=$ $0.5, d_{2}=10$ and $p=0.07$.

Summarizing the numerical results exhibited in Figs. $8-12$, we claim that a fact that the connection probability $p$ has a essential influence on the pattern formation in the random networked system. This is very different from the pattern formation in the continuous media systems. Precisely, for the continuous media system, if the parameters are fixed in the Turing instability region, then the eigenvalues of the Laplacian matrix are fixed. Hence, the Turing pattern may exist. However, for the random network system, although the parameters are fixed in the Turing instability, the eigenvalues of the discrete Laplacian matrix, $\Lambda_{i}$, are not fixed, namely it may be in or out of the Turing instability area with the change of the connection probability $p$. Thence, apart from diffusion coefficients, the connection probability $p$ also has a crucial influence on the pattern formation of random network systems compared with the continuous media systems.
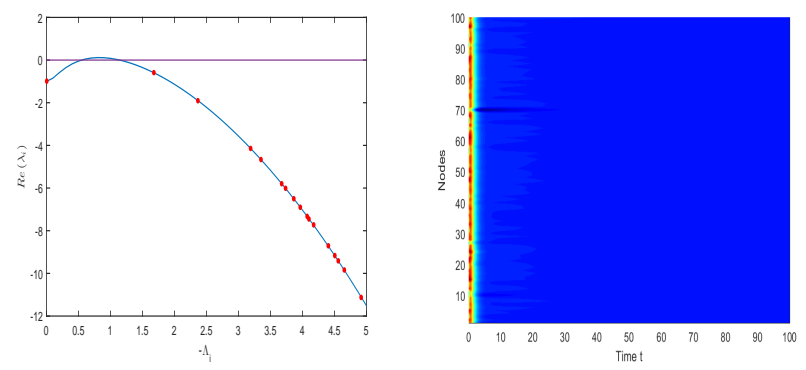

Fig. 12 There is no eigenvalues of the discrete Laplacian matrix locate in Turing instability region, and positive equilibrium $E_{*}$ becomes stable again. Here parameters are $c=$ $2.3, \beta=1, d_{1}=0.5, d_{2}=10$ and $p=0.09$.

\section{Conclusions}

In this work, a general activator-substrate model with network structure is introduced to understand the formation of spatiotemporal patterns. We first consider the general continuous activator-substrate model, here the maximum principle and the Harnack's inequality are used to investigate the boundedness of the nonconstant steady state of the corresponds elliptic system. It is found that the non-constant steady state admits their upper and lower bounds with certain conditions, see Theorem 1. Then, the Poincaré's inequality, Cauchy-Schwarz inequality and the technique of integrating by parts are employed to research some properties and non-existence of the non-constant steady state with the no-flux boundary conditions. One shows that the general activator-substrate model admits the nonconstant steady state if the diffusion rate of activator greater than the diffusion rate of substrate, see Theorem 2 and Theorem 3. Afterwards, a general random network activator-substrate model is introduced. The existence conditions of the stability, the Hopf bifurcation, the Turing instability and the Turing-Hopf bifurcation are yield by the method of linear analysis and bifurcation theorem, see Theorems 4-6, respectively. Finally, a suitable sub-system of the general activatorsubstrate model is investigated, and full numerical simulations are well verified these results. Especially, the unstable positive equilibrium $E_{*}$ will become stable with the change of the connection probability $p$ by comparing Figs. 8-12. Hence, the connection probability $p$ of the nodes is also an important factor for the formation of the Turing pattern compared with the continuous media systems. In addition, whether the degree of nodes has an influence on the pattern formation in the random network systems also is worth exploring. We will research it in future works.

\section{Declaration}

\section{Funding}

This work was supported by the National Natural Science Foundation of China (Nos. 11971032, 62073114), Ranchao Wu and Liping Chen is supported by these two foundations (Nos. 11971032, 62073114), and Mengxin Chen is supported by the foundation (No. 11971032), Qianqian Zheng is supported by Young Talent Support Project of Henan (2020HYTP012). 


\section{Compliance with ethical standards}

The work complies with ethical standards.

\section{Conflicts of interest/Competing interests}

The authors declare that they have no conflict of interest.

\section{Availability of data and material}

All data and material generated or analysed during this study are included in this published article.

\section{References}

1. Chen, M.X., Wu, R.C., Chen, L.P.: Spatiotemporal patterns induced by Turing and Turing-Hopf bifurcations in a predator-prey system, Appl. Math. Comput. 380, 125300 (2020).

2. Wang, J.F., Wu, S.N., Shi, J.P.: Pattern formation in diffusive preydator-prey systems with predator-taxis and prey-taxis, Discret. Cont. Dyn. Syst. B 26(3), 1273-1289 (2021).

3. Wang, W., Liu, S.T., Tian, D.D., et al.: Pattern dynamics in a toxin-producing phytoplankton-zooplankton model with additional food, Nonlinear Dyn. 94(1), 211228 (2018).

4. Berenstein, I.: Standing wave-like patterns in the GrayScott model, Chaos 25(6), 064301 (2015).

5. Chen, M.X., Wu, R.C., Liu, B., Chen, L.P.: Hopf-Hopf bifurcation in the delayed nutrient-microorganism model, Appl. Math. Model. 86, 460-483 (2020) .

6. Tan, W., Yu, W.W., Hayat, T., et al.: Turing instability and bifurcation in a diffusion predator-prey model with Beddington-DeAngelis functional response, Int. J. Bifurcat. Chaos 28(9), 1830029 (2018).

7. Pal, S., Petrovskii, S., Ghorai, S., Banerjee, M.: Spatiotemporal pattern formation in $2 \mathrm{D}$ prey-predator system with nonlocal intraspecific competition, Commun. Nonlinear Sci. Numer. Simul. 93, 105478 (2021).

8. Chen, M.X., Wu, R.C., Chen, L.P.: Pattern dynamics in a diffusive Gierer-Meinhardt model, Int. J. Bifurcat. Chaos 30(12), 2030035 (2020).

9. Song, Y.L., Jiang, H.P., Yuan, Y.: Turing-Hopf bifurcation in the reaction-diffusion system with delay and application to a diffusive predator-prey model, J. Appl. Anal. Comput. 9(3), 1132-1164 (2019).

10. Chen, M.X., Wu, R.C., Liu, B., et al.: Spatiotemporal dynamics in a ratio-dependent predator-prey model with time delay near the Turing-Hopf bifurcation point, Commun. Nonlinear Sci. Numer. Simul. 77, 141-167 (2019).

11. Asllani M., Challenger, J.D., Pavone F.S., et al.: The theory of pattern formation on directed networks, Nat. Conmmun. 5(1), 1-9 (2014).

12. Diego, X., Marcon, L., Müller, P., Sharpe, J.: Key features of Turing systems are determined purely by network topology, Phys. Rev. X 8, 021071 (2018).

13. Chang, L.L., Liu, C., Sun, G.Q., et al.: Delay-induced patterns in a predator-prey model on complex networks with diffusion, New J. Phys. 21, 073035 (2019).
14. Tian, C.R., Ling, Z., Zhang, L.: Delay-driven spatial patterns in a network-organized semiarid vegetation model, Appl. Math. Comput. 367, 124778 (2020).

15. Mimar, S., Juane, M.M., Park, J., et al.: Turing patterns mediated by network topology in homogeneous active systems, Phys. Rev. E 99(6), 062303 (2019).

16. Nakao, H., Mikhailov, A.S.: Turing patterns in networkorganized activator-inhibitor systems. Nat. Phys. 6, 544$550(2010)$.

17. Wyller, J., Blomquist, P., Einevoll, G.T.: Turing instability and pattern formation in a two-population neuronal network model. Phys. D 225, 75-93 (2007)

18. Tian, C.R., Ruan, S.G.: Pattern formation and synchronism in an allelopathic plankton model with delay in a network, SIAM J. Appl. Dyn. Syst. 18(1), 531-557 (2019).

19. Zheng, Q.Q., Shen, J.W.: Turing instability induced by random network in FitzHugh-Nagumo model, Appl. Math. Comput. 381, 125304 (2020).

20. Gierer, A., Meinhardt, H.: A theory of biological pattern formation, Kybernetik 12, 30-39 (1972).

21. Meinhardt, H., Klingler, M.: A model for pattern formation on the shells of molluscs, J. Theor. Biol. 126, 63-89 (1987).

22. Fowlery, D.R., Meinhardtz, H., Prusinkiewicz, P.: Modeling seashells, Comput. Graph. 26(2), 379-387 (1992).

23. Wu, R.C., Shao, Y., Zhou, Y., Chen, L.P.: Turing and Hopf bifurcation of Giere-Meinhardt activator-substrate model, Electron. J. Differ. Equations 173, 1-19 (2017).

24. Wang, Y.E., Wu, J.H., Jia, Y.F.: Steady-state bifurcation for a biological depletion model, Int. J. Bifurcat. Chaos 26(4), 1650066 (2016).

25. Kelkel, J. Surulescu, C.: A weak solution approach to a reaction-diffusion system modeling pattern formation on seashells, Math. Meth. Appl. Sci. 32, 2267-2286 (2009).

26. Lou, Y., Ni, W.M.: Diffusion, self-diffusion and crossdiffusion, J. Differ. Equations 131(1), 79-131 (1996).

27. Lin, C.S., Ni, W.M., Takagi, I.: Large amplitude stationary solutions to a chemotaxis system, J. Differ. Equations, 72(1), 1-27 (1988).

28. Kelkel, J., Surulescu, C.: On a stochastic reactiondiffusion system modeling pattern formation on seashells, J. Math. Biol. 60, 765-796 (2010).

29. Watts, D.J., Strogatz, S.H.: Collective dynamics of smallworld networks, Nature 393, 440-442 (1998). 